\title{
Identification and characterization of a Streptococcus equi ssp. zooepidemicus immunogenic GroEL protein involved in biofilm formation
}

Li Yi ${ }^{1,2}$, Yang Wang ${ }^{1,3}$, Zhe Ma ${ }^{1}$, Hui-Xing Lin ${ }^{1}$, Bin Xu ${ }^{1}$, Daniel Grenier ${ }^{4}$, Hong-Jie Fan ${ }^{1,5^{*}}$ and Cheng-Ping Lu ${ }^{1}$

\begin{abstract}
Streptococcus equi ssp. zooepidemicus (S. equi spp. zooepidemicus) is an opportunistic pathogen that causes major economic losses in the swine industry in China and is also a threat for human health. Biofilm formation by this bacterium has been previously reported. In this study, we used an immunoproteomic approach to search for immunogenic proteins expressed by biofilm-grown S. equi spp. zooepidemicus. Seventeen immunoreactive proteins were found, of which nine common immunoreactive proteins were identified in planktonic and biofilm-grown bacteria. The immunogenicity and protective efficacy of the S. equi spp. zooepidemicus immunoreactive GroEL chaperone protein was further investigated in mice. The protein was expressed in vivo and elicited high antibody titers following S. equi spp. zooepidemicus infections of mice. An animal challenge experiment with S. equi spp. zooepidemicus showed that 75\% of mice immunized with the GroEL protein were protected. Using in vitro biofilm inhibition assays, evidence was obtained that the chaperonin GroEL may represent a promising target for the prevention and treatment of persistent S. equi spp. zooepidemicus biofilm infections. In summary, our results suggest that the recombinant GroEL protein, which is involved in biofilm formation, may efficiently stimulate an immune response, which protects against $S$. equi spp. zooepidemicus infections. It may therefore be a candidate of interest to be included in vaccines against $S$. equi spp. zooepidemicus infections.
\end{abstract}

\section{Introduction}

Streptococcus equi ssp. zooepidemicus (S. equi spp. zooepidemicus) is an opportunistic pathogen that causes important economic losses in the swine industry in China and is also a threat for human health [1]. It is indeed a major problem in China and the development of an effective vaccine is vital to circumvent the significant economic impact to the industry. Previous studies have investigated different immunogenic proteins of planktonically grown $S$. equi spp. zooepidemicus, including secreted and surface-associated proteins [2-4]. Mao et al. $[3,4]$ used the immunoproteomic technology to

\footnotetext{
*Correspondence: fhj@njau.edu.cn

${ }^{1}$ Key Lab of Animal Bacteriology, Ministry of Agriculture, Nanjing Agricultural University, Nanjing, China

Full list of author information is available at the end of the article
}

study the convalescent serum of mini-pigs and identified 12 membrane-associated proteins, 13 cell wall-associated proteins, and seven extracellular proteins in S. equi spp. zooepidemicus. Based on enzymatic shaving and Western blot analyses, Wei et al. [2] described five novel membrane-associated vaccine candidate proteins.

We recently reported that $S$. equi spp. zooepidemicus can form biofilms [5]. Given that bacteria within biofilms have an increased resistance to the host defence system and antibiotics, infections caused by biofilm-producing bacteria are frequently chronic, recurrent, and resistant to antibacterial chemotherapy [6]. Immunoprophylaxis and immunotherapy targeting biofilm-related proteins are promising new approaches for the prevention and treatment of biofilm-associated infections [7]. The immunoproteomic technology has been successfully used to identify relevant bacterial antigens for the development 
of new vaccines $[8,9]$. This technique might also be useful to increase our understanding of the molecular mechanisms that control biofilm formation by $S$. equi spp. zooepidemicus.

In the present study, immunogenic proteins expressed by biofilm-grown $S$. equi spp. zooepidemicus were identified using an immunoproteomic approach. The chaperonin GroEL was found to be an immunogenic protein in both biofilm- and planktonic-grown S. equi spp. zooepidemicus. The immunogenicity of the recombinant GroEL protein, the protection rate of GroEL-immunized mice, and the capacity of anti-GroEL antibodies to inhibit biofilm production by $S$. equi spp. zooepidemicus in vitro were also investigated.

\section{Materials and methods}

\section{Ethical statements}

All the animal experiments were performed with the approval of the Laboratory Animal Monitoring Committee of Jiangsu Province (SYXK (SU) 2011-0036). All efforts were made to minimize animal suffering and to reduce the number of animals used.

\section{Bacteria and culture conditions}

Streptococcus equi ssp. zooepidemicus strain ATCC 35246 was initially isolated from a diseased pig in Sichuan Province, China, in 1976. The strain, which was purchased from the American Type Culture Collection (ATCC), was cultured in Todd-Hewitt broth (THB, Oxoid) and on THB agar plates. Biofilms of S. equi spp. zooepidemicus were prepared by growing bacteria in THB medium supplemented with $1 \%$ human fibrinogen (Sigma) in polystyrene Petri dishes at $37^{\circ} \mathrm{C}$ for $24 \mathrm{~h}$, as described previously [5]. Quantification of biofilm production was carried out by a microtiter plate assay using crystal violet staining. $S$. equi spp. zooepidemicus planktonic cells grown at $37^{\circ} \mathrm{C}$ for $24 \mathrm{~h}$ in Erlenmeyer flasks containing the above culture medium were used as the control.

\section{Whole cell protein sample extraction}

Bacterial proteins were prepared as described previously $[5,10]$. Briefly, following biofilm formation, the medium containing free-floating bacteria was poured off, and the wells were washed three times with sterile PBS to remove loosely attached bacteria. The biofilms were detached by scraping, were suspended in $50 \mathrm{mM}$ Tris- $\mathrm{HC} 1$ (pH 7.5), and were sonicated for $5 \mathrm{~min}$. The sonicated biofilms were pelleted by centrifugation at $8000 \times g$ for $5 \mathrm{~min}$ at $4{ }^{\circ} \mathrm{C}$. The pellets were washed twice in $50 \mathrm{mM}$ Tris- $\mathrm{HC} 1$ (pH 7.5) and were then resuspended in buffer $(50 \mathrm{mM}$ Tris- $\mathrm{HCl}, \mathrm{pH} 7.5,3 \mathrm{mM} \mathrm{MgCl}, 50 \%$ sucrose) containing $1000 \mathrm{U} / \mathrm{mL}$ of mutanolysin (Sigma) and were incubated for $90 \mathrm{~min}$ at $37^{\circ} \mathrm{C}$. Spheroplasts were collected and were resuspended by sonication on ice $(100 \mathrm{~W}$, 90 cycles, $5 \mathrm{~s}$ on and $10 \mathrm{~s}$ off $)$ in sonication buffer $(7 \mathrm{M}$ urea, $2 \mathrm{M}$ thiourea, 4\% 3-[(3-cholamidopropyl) dimethylammonio]-1- propanesulfonate (CHAPS), and $65 \mathrm{mM}$ dithiothreitol (DTT,GE Healthcare). Spheroplasts were then incubated at $25{ }^{\circ} \mathrm{C}$ for $30 \mathrm{~min}$, following which cell debris and unbroken cells were removed by centrifugation at $10000 \times g$ for $30 \mathrm{~min}$ at $25^{\circ} \mathrm{C}$. The supernatants were mixed with $10 \%$ Trichloroacetic acid (TCA) and were incubated in ice water for $30 \mathrm{~min}$. The precipitated proteins were pelleted by centrifugation at $10000 \times g$ for $10 \mathrm{~min}$ at $4{ }^{\circ} \mathrm{C}$ and were washed twice with pre-chilled acetone. The final pellet was air-dried, dissolved in sample preparation solution (7 M urea, $2 \mathrm{M}$ thiourea, $4 \%$ CHAPS, $65 \mathrm{mM} \mathrm{DTT}$ ), incubated for $30 \mathrm{~min}$ at $25{ }^{\circ} \mathrm{C}$ (vortexed every $10 \mathrm{~min}$ ), and centrifuged at $10000 \times g$ for $20 \mathrm{~min}$ at $25{ }^{\circ} \mathrm{C}$. Prior to rehydration, the supernatants were treated with 2-D Clean-up kits (GE Healthcare) to remove contaminants that may interfere with the electrophoresis. The protein content was determined using PlusOne 2-D Quant kits (GE Healthcare).

\section{2-D gel electrophoresis}

The 2-DE (two-dimensional gel electrophoresis) was performed using the immobiline/polyacrylamide system. The isoelectric focusing (IEF) was performed using IPG DryStrips (13 cm; IPGphor; GE Healthcare). Protein samples $(200 \mu \mathrm{g})$ were applied to the IPG strips using the in-gel sample rehydration technique according to the manufacturer's instructions. The IEF was performed in a protein IEF cell (GE Healthcare) using a stepwise voltage gradient to $80 \mathrm{kVh}$. The strips were equilibrated for $2 \times 15 \mathrm{~min}$ in equilibration buffer (6 M urea, $2 \%$ SDS, $30 \%$ glycerol, $50 \mathrm{mM}$ Tris $-\mathrm{HCl}, \mathrm{pH} 8.8$ ) supplemented with $1 \%$ DTT and $4 \%$ iodoacetamide prior to running the second dimension. The SDS-PAGE was carried out vertically on $12.5 \%$ polyacrylamide gels using an Ettan DALT II system (GE Healthcare). Resolved proteins were routinely stained with Coomassie Brilliant Blue G-250 for protein identification purposes. All experiments were performed in triplicate. The reproducibility of the 2-DE was verified by running the same samples at least three times on separate gels. Three replicate gels from three independent experiments were run for each growth condition. The gels were compared using Image Master Platinum 5.0 software (GE Healthcare).

\section{Preparation of convalescent sera}

The pathogen-free mini-pigs used in this study had no history of S. equi spp. zooepidemicus infection and were found to be negative for antibodies against $S$. equi spp. zooepidemicus ATCC 35246 whole cells, as determined by an enzyme-linked immunosorbent assay (ELISA). 
Swine convalescent sera were obtained from the pigs artificially infected with live $S$. equi spp. zooepidemicus ATCC 35246. Pre-infection sera were used as a negative control. The protocol of Zhang and Lu [11] was used for the immunization procedure and immunogen preparation. Pigs were intramuscularly injected twice at 3-week interval with $1.0 \times 10^{9} \mathrm{CFU}$ (Colony-Forming Units) of S. equi spp. zooepidemicus. Swine sera were collected 7 days after the booster injection, and serum IgG antibody titers were determined using a whole cell ELISA [3]. Wells of the microplate were coated with formaldehydeinactive $S$. equi spp. zooepidemicus $\left(1.0 \times 10^{6}\right.$ cells $)$, and blocked with $5 \%$ skim milk. Two-fold serial dilutions (from 1:500 to 1:16 000) of the sera were added and the plate was incubated for $2 \mathrm{~h}$. Following incubation in the presence of horseradish peroxidase (HRP)-conjugated goat anti-mouse IgG as the secondary antibody, the color was developed by adding 3,3',5,5'-tetramethylbenzidine (TMB) substrate (Beyotime Institute, China). Color development was recorded by reading the absorbance of $450 \mathrm{~nm}$ using a microtiter plate reader (Bio-Rad, USA). Sera with high titers were selected for subsequent experiments.

\section{Western blotting}

Protein samples from each SDS-PAGE gel were transferred onto polyvinylidene difluoride (PVDF) membranes (GE Healthcare) using a semi-dry blotting apparatus (TE77, GE Healthcare) for $2 \mathrm{~h}$ at $0.65 \mathrm{~mA} / \mathrm{cm}^{2}$. After the transfer, membranes were blocked by incubation for $2 \mathrm{~h}$ in $100 \mathrm{mM}$ Tris- $\mathrm{HCl}, 150 \mathrm{mM} \mathrm{NaCl}$, and $0.05 \%$ Tween-20 (TBST) containing 5\% skim milk. The blocked membranes were incubated with sera from convalescent mini-pigs (1:1000 dilution) for $2 \mathrm{~h}$ at room temperature with gentle agitation. They were then washed three times with TBST (10 min per wash) and were incubated ( $1 \mathrm{~h}$ with gentle agitation) with horseradish peroxidase-labeled Staphylococcal protein A (Boster, China) at a dilution of 1:5000 in blocking solution. The membranes were washed as before, and were incubated with 3,3'-diaminobenzidine (Tiangen, China) until the optimum color was obtained. This analysis was repeated three times for each sample.

\section{Mass spectrometric analysis of protein spots and database searches}

The Coomassie Brilliant Blue-stained spots corresponding to the immunoreactive proteins were excised from the 2-D gels and were sent to Shanghai Applied Protein Technology Co. Ltd for tryptic in-gel digestion, Matrix-Assisted Laser Desorption/Ionization Time of Flight Mass Spectrometry (MALDI-TOF-MS), and MALDI-TOF/TOF-MS. The MALDI-TOF-MS and
MALDI-TOF/TOF-MS acquisition data were used in a combined search against the NCBInr protein database using MASCOT (Matrix Science), with parameter sets for trypsin digestion, one max missed cleavage, variable modification of oxidation (M), and a peptide mass tolerance for monoisotopic data of $100 \mathrm{ppm}$. The MASCOT server was originally used to search the NCBInr for peptide mass fingerprinting (PMF). The criteria used to accept protein identifications were based on PMF data, including the extent of sequence coverage, the number of peptides matched, and the score of probability. Protein identification was assigned when the following criteria were met: at least four matching peptides and sequence coverage greater than $15 \%$.

\section{Confirmation of the immunogenicity of selected proteins}

The GroEL chaperone protein was selected for molecular cloning and its immunogenicity was confirmed. The primer pairs of GroEL-S (5'-CGCGAATTCGATA TTTTGGCGGATACCGT-3') and GroEL-A (5'-CC CTCGAGAGCAGGCTCTGGCTTAGTGG-3') were designed to express the recombinant protein based on the gi number (Table 1). More specifically, GroEL PCR product was cloned into the pET28a expression vector as described in a previous study [12]. The resulting plasmid was used to transform Escherichia coli DH5 $\alpha$. (TaKaRa, China). Expression of the recombinant protein was induced in E. coli BL21(DE3) (TaKaRa, China) at $37{ }^{\circ} \mathrm{C}$ for $4 \mathrm{~h}$ with $0.1 \mathrm{mM}$ IPTG (Isopropyl $\beta$-DThiogalactoside). The protein was purified by HisTrap FF column (GE Healthcare, USA) and purity was analyzed by SDS-PAGE [13]. The immunogenicity of the GroEL protein was confirmed by Western blot analysis using pig convalescent sera directed against the $S$. equi spp. zooepidemicus ATCC 35246 strain.

\section{Mouse vaccination and determination of protection efficacy}

Thirty-two ICR (Institute of Cancer Research) mice (4-week-old females) were randomly assigned to four groups of eight mice each. The mice in Group 1 were intramuscularly injected with $0.2 \mathrm{~mL}$ of recombinant GroEL protein (rGroEL; $100 \mu \mathrm{g} / \mathrm{mL}$ ) mixed with an equivalent volume of MONTANIDE ISA 206 VG (SEPPIC, France). The mice in Group 2 were intramuscularly injected with $0.2 \mathrm{~mL}$ of formaldehyde-inactivated S. equi spp. zooepidemicus ATCC35246 vaccine $\left(1 \times 10^{9} \mathrm{cfu} / \mathrm{mL}\right)$ combined with MONTANIDE ISA 206 VG and served as positive controls. The second booster from groups 1 and 2 were intramuscularly injected with the recombinant GroEL protein and whole-cell vaccine at day 14 , respectively. The mice in group 3 were intramuscularly injected with PBS and served as negative controls. The 
Table 1 Immunoproteins identified by MALDI-TOF/TOF MS

\begin{tabular}{|c|c|c|c|c|c|c|c|}
\hline Spot no. & $\begin{array}{l}\text { Protein } \\
\text { identified }^{\text {a }}\end{array}$ & $\begin{array}{l}\text { BLASTX similarity } \\
\text { matched protein/ } \\
\text { species/identity } \\
\text { score }\end{array}$ & $\begin{array}{l}\text { Theoretical MW } \\
/ \mathrm{pl}^{\mathrm{b}}\end{array}$ & $\begin{array}{l}\text { Experimental } \\
\mathrm{MW} / \mathrm{pl}\end{array}$ & MASCOT score ${ }^{c}$ & $\begin{array}{l}\text { No. of peptides } \\
\text { matched }^{d}\end{array}$ & Coverage (\%) \\
\hline BF1 & gi|338846659 & $\begin{array}{l}\text { DNA polymerase III } \\
\text { delta subunit }\end{array}$ & $39994 / 5.80$ & $22000 / 5.70$ & 252 & 19 & 55 \\
\hline $\mathrm{BF} 2$ & gi|195977415 & elongation factor $\mathrm{G}$ & $76540 / 4.83$ & $76000 / 4.80$ & 232 & 20 & 40 \\
\hline $\mathrm{BF} 3$ & gi|195978009 & pyruvate kinase & $54638 / 5.09$ & $55000 / 5.10$ & 374 & 36 & 62 \\
\hline BF4 & gi|338847723 & Transketolase & $71341 / 5.07$ & $71000 / 5.09$ & 272 & 11 & 21 \\
\hline BF5 & gi|338846410 & $\begin{array}{l}\text { chaperone protein } \\
\text { DnaK (heat shock } \\
\text { protein 70) }\end{array}$ & 65 045/4.64 & $60000 / 4.60$ & 269 & 22 & 36 \\
\hline BF6 & gi|225867742 & $\begin{array}{l}60 \text { kDa chaperonin } \\
\text { GroEL }\end{array}$ & $56876 / 4.70$ & $56000 / 4.60$ & 575 & 34 & 60 \\
\hline BF7 & gi|195978262 & $\begin{array}{l}30 \text { ribosomal } \\
\text { protein S1 }\end{array}$ & $43801 / 4.93$ & 43 000/5.00 & 172 & 16 & 46 \\
\hline BF8 & gi|338848099 & $\begin{array}{l}\text { inosine-5'- } \\
\text { monophosphate } \\
\text { dehydrogenase }\end{array}$ & $53007 / 5.48$ & $53000 / 5.60$ & 257 & 29 & 63 \\
\hline BF9 & gi|338846419 & $\begin{array}{l}\text { 3-oxoacyl-(acyl- } \\
\text { carrier-protein) } \\
\text { synthase II }\end{array}$ & $43738 / 5.44$ & $40000 / 5.40$ & 164 & 17 & 52 \\
\hline BF10 & gi|338847987 & $\begin{array}{l}\text { adenylosuccinate } \\
\text { synthetase }\end{array}$ & $47501 / 5.47$ & $47000 / 5.60$ & 155 & 18 & 49 \\
\hline BF11 & gi|225869251 & $\begin{array}{l}\text { phosphoglycerate } \\
\text { kinase }\end{array}$ & $42184 / 4.96$ & $42000 / 4.90$ & 120 & 14 & 38 \\
\hline BF12 & gi|225867788 & $\begin{array}{l}\text { glucose-6-phos- } \\
\text { phate isomerase }\end{array}$ & 49 490/4.88 & $42000 / 4.80$ & 207 & 23 & 48 \\
\hline BF13 & gi|225869252 & $\begin{array}{l}\text { glyceraldehyde- } \\
\text { 3-phosphate } \\
\text { dehydrogenase }\end{array}$ & $37025 / 5.57$ & $40000 / 5.00$ & 243 & 24 & 58 \\
\hline BF14 & gi|338846346 & $\begin{array}{l}\text { pyridine nucleo- } \\
\text { tide-disulfide } \\
\text { oxidoreductase }\end{array}$ & $47715 / 5.30$ & $47000 / 5.30$ & 191 & 21 & 56 \\
\hline BF15 & gi|338847824 & $\begin{array}{l}\text { oligopeptide ABC } \\
\text { transporter } \\
\text { periplasmic } \\
\text { oligopeptide- } \\
\text { binding protein } \\
\text { OppA }\end{array}$ & $72742 / 5.56$ & $72000 / 5.60$ & 244 & 25 & 49 \\
\hline BF16 & gi|338847405 & $\begin{array}{l}\text { Elongation factor- } \\
\text { Tu }\end{array}$ & $44545 / 4.89$ & $21000 / 5.30$ & 109 & 9 & 22 \\
\hline BF17 & gi|225867729 & $\begin{array}{l}\text { elongation factor } \\
\text { Ts }\end{array}$ & 37 263/4.86 & $30000 / 4.40$ & 193 & 20 & 59 \\
\hline
\end{tabular}

a gi number in NCBI.

b Theoretical MW and pl was calculated using compute pl/MW [38].

c MASCOT score obtained for the peptide mass fingerprint (PMF). The significance threshold was 70 .

d Number of peptides that match the predicted protein sequence.

e Percentage of predicted protein sequence covered by matched peptides.

mice in Group 4 served as blank controls. One hundred $\mu \mathrm{L}$ of orbit blood were collected from each mouse. The blood samples used to prepare the sera were obtained on days $0,7,14,24$, and 28 . Three mice from each group were sampled at random on each date. Blood were clotted and sera were prepared by centrifugation and were stored at $-20^{\circ} \mathrm{C}$ for later analysis. To measure serum IgG antibody, all titers of sera were evaluated using indirect ELISA.

Two weeks after the second booster dose, the mice in groups 1-3 were inoculated intraperitoneally with $0.2 \mathrm{~mL}$ of bacterial suspensions $\left(1.25 \times 10^{6} \mathrm{cfu} / \mathrm{mL}\right.$, approximately $5 \times \mathrm{LD}_{50}$ ) obtained from an 18-h culture in THB to evaluate the protection against an $S$. equi spp. 
zooepidemicus challenge $[14,15]$. Another eight mice in Group 4 were injected with $0.2 \mathrm{~mL}$ of sterile PBS.

\section{The effect of anti-rGroEL antibodies on biofilm formation by S. equi spp. zooepidemicus}

The biofilm assay was performed as described by Zarankiewicz et al. [16], with modifications. A S. equi spp. zooepidemicus colony was inoculated into $5 \mathrm{~mL}$ of THB and was cultivated with shaking at $37{ }^{\circ} \mathrm{C}$ for $12 \mathrm{~h}$ (late exponential/stationary growth phase) [15]. The culture was diluted 1:100 with fresh THB broth supplemented with $1 \%$ fibrinogen and with either $1 \%$ pooled mouse anti-rGroEL serum or $1 \%$ pooled non-immunized mouse sera from day 35 . After a $2 \mathrm{~h}$ incubation at $4{ }^{\circ} \mathrm{C}, 200 \mu \mathrm{L}$ of the mixtures $\left(10^{6}\right.$ cell per well) was added to each well of a 96-well polystyrene microtiter plate. The plate was incubated at $37{ }^{\circ} \mathrm{C}$ for $24 \mathrm{~h}$ without shaking. A S. equi spp. zooepidemicus culture in THB medium containing $1 \%$ fibrinogen was used for the positive control. Uninoculated culture medium containing $1 \%$ fibrinogen was used for the negative control. Biofilms were quantified by crystal violet staining as described by Wang et al. [17]. The medium containing free-floating bacteria was poured off and the wells were washed three times with sterile PBS. Microtiter plate wells were then stained with $200 \mu \mathrm{L}$ of $1 \%(\mathrm{w} / \mathrm{v})$ crystal violet for $10 \mathrm{~min}$ and washed four times with PBS to remove unbound crystal violet dye, and then air dried for $1 \mathrm{~h}$. Biofilm-adsorbed crystal violet was resolubilized by adding $200 \mu \mathrm{L} 95 \%$ (v/v) ethanol of each well, and the absorbance was measured at $595 \mathrm{~nm}$. All the assays were performed in triplicate.

\section{Statistical analysis}

Statistical analyses were carried out using the GraphPad Software package. Survival data were assessed by Kaplan C Meier survival analysis and tested for significance by the $\log$ rank test. Other data were analyzed using the Student's $t$ test and the $P$ values $<0.05$ were considered significant.

\section{Results}

\section{Identification of immunoreactive proteins}

A 2-DE covering a $\mathrm{pH}$ range of 4-7 (IPG linear gradient) was performed to separate whole cell proteins prepared from biofilm-grown S. equi spp. zooepidemicus. The spots were detected by Coomassie Brilliant Blue G-250 staining (Figure 1A). Western blotting with pig convalescent sera revealed the presence of seventeen immunoreactive proteins in the biofilm-grown bacterial samples (Figure 1B), which was consistent with our observations of the duplicated 2-D gel (Figure 1A). The seventeen spots were excised and were characterized by MALDITOF-MS and MALDI-TOF-TOF-MS, and the data were compared to those in the NCBI sequence database. The probability score for the match, MW, pI, and number of peptide matches were used to identify the spots. The seventeen immunoreactive spots, listed in Table 1, corresponded to seventeen different proteins. Nine of these immunoreactive proteins were also identified in planktonic-grown bacteria using the same protocol [4]: a DNA polymerase III delta subunit, elongation factor G, pyruvate kinase, transketolase, the GroEL molecular chaperone, glyceraldehyde-3-phosphate dehydrogenase, pyridine nucleotide-disulfide oxidoreductase, oligopeptide $\mathrm{ABC}$ transporter periplasmic oligopeptide-binding protein OppA, and elongation factor Ts. Since the GroEL molecular chaperone is essential for biofilm formation in other bacteria [18], it was selected for further analysis.

\section{Western blot analysis of the recombinant proteins}

The GroEL protein of S. equi spp. zooepidemicus was cloned in E. coli. The immunogenicity of the rGroEL protein was confirmed by Western blotting using mini-pig convalescent sera (Figure 2).

\section{Antibody response to vaccination with the rGroEL protein} The GroEL-specific antibody response elicited by immunization with the rGroEL protein was monitored by determining the serum antibody titers of all the experimental mice. The GroEL protein-specific antibody titers of mice vaccinated with the recombinant protein were markedly higher than those of the PBS-injected mice at day 7 post-vaccination and continued to increase by day 28 (Figure 3). The antibody titers of the rGroEL proteinvaccinated mice were significantly higher $(P<0.01)$ than those of the PBS-injected mice at all time points postvaccination. No significant differences in titers were found between the rGroEL protein-vaccine and the inactivated $S$. equi spp. zooepidemicus vaccine $(P>0.05)$.

\section{Immunoprotection provided by the rGroEL vaccine in mice} Immunized and non-immunized mice were monitored daily for 7 days following a challenge with $S$. equi spp. zooepidemicus. In the non-immunized group, the first death of mice occurred $24 \mathrm{~h}$ after the challenge, and the mortality rate reached $100 \%$ within $48 \mathrm{~h}$. In the groups immunized with rGroEL or the inactivated bacterial vaccine, only 2 out of 8 mice (25\%) died following the challenge with S. equi spp. zooepidemicus. More specifically, the deaths occurred at day 3 post-injection. The immunoprotection rate for these two groups was $75 \%$ in both cases (Figure 4). Compared with the non-immunized group,the mice in the groups immunized with rGroEL or the inactivated $S$. equi spp. zooepidemicus vaccine had higher survival rates $(P<0.05)$. No significant differences in survival rates were found between the group 

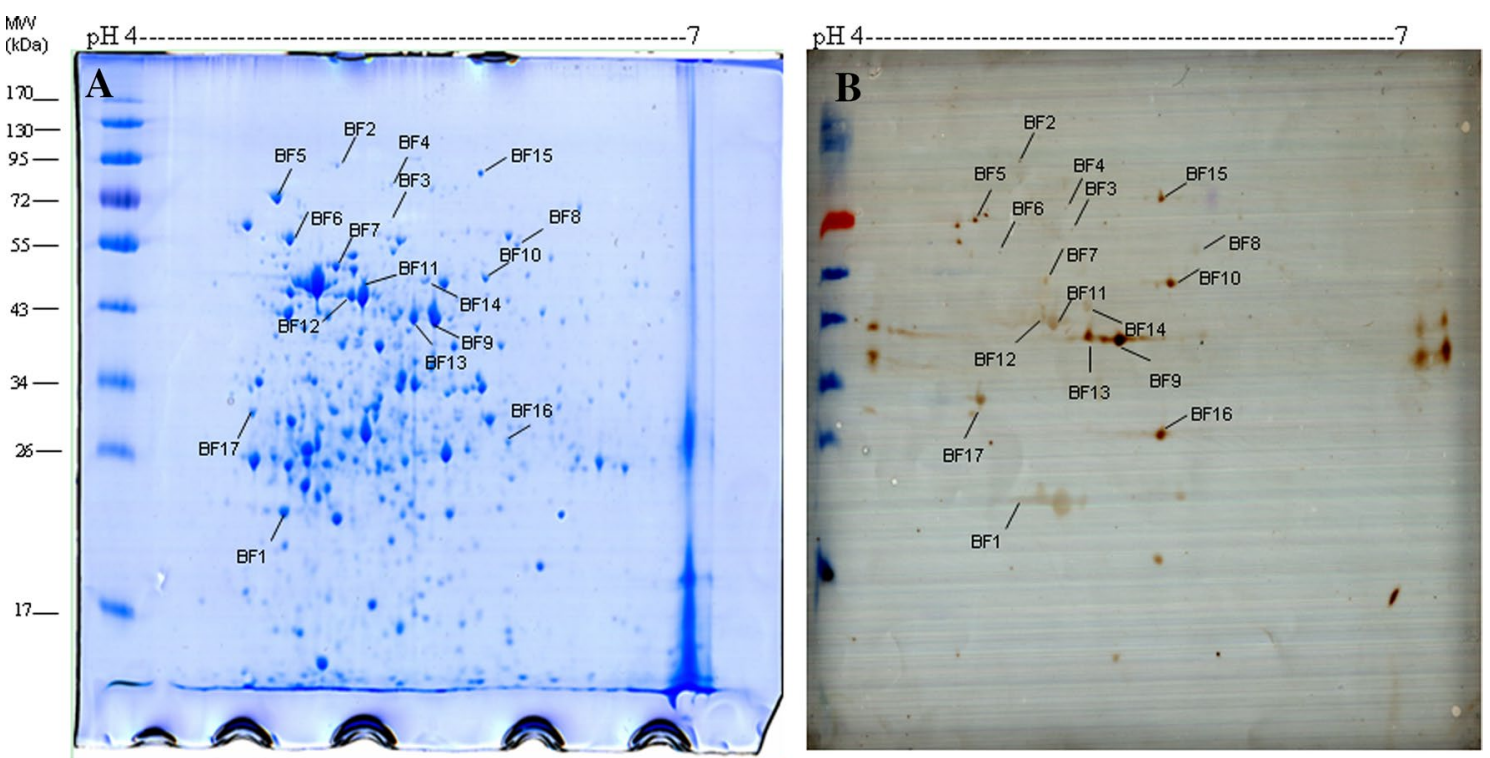

Figure 1 2-D gel electrophoresis profiles of whole cell lysates of biofilm-grown S. equi spp. zooepidemicus with the immunoreactive proteins indicated. A Protein staining with Coomassie B-250. B Western blot analysis of the immunoreactive proteins using pig convalescent serum. Immunoreactive spots are indicated by the abbreviation of biofilm (BF) followed by an arbitrary number.



immunized with rGroEL and the group inactivated $S$. equi spp. zooepidemicus vaccine $(P>0.05)$.

\section{Biofilm inhibition by the anti-rGroEL antibody in vitro}

Biofilm formation by $S$. equi spp. zooepidemicus was assessed in a microplate assay and crystal violet staining. Biofilm formation by S. equi spp. zooepidemicus cultured in THB medium supplemented with anti-rGroEL

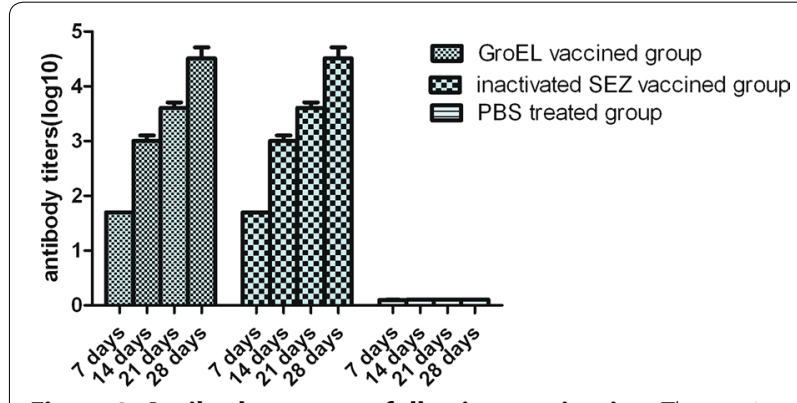

Figure 3 Antibody response following vaccination. Three mice from each group were sampled at random on each date. Data represent the mean \pm standard deviation ( $n=3$ per group) of antibody titers (log 10) vs. days post-vaccination. Bars indicate standard deviations. The antibody titers of the rGroEL protein-vaccinated mice were significantly higher $(P<0.01)$ than those of the PBS-injected mice at all time points post-vaccination. No significant differences in titers were found between the rGroEL protein-vaccine and the inactivated S. equi spp. zooepidemicus vaccine $(P>0.05)$.

serum $(0.32 \pm 0.06)$ was significantly lower $(P<0.01)$ than that of S. equi spp. zooepidemicus cultured in THB medium alone $(1.11 \pm 0.05)$ or in THB medium supplemented with non-immunized pathogen-free mouse serum $(0.96 \pm 0.10)$. No significant differences in $\mathrm{A}_{595 \mathrm{~nm}}$ were found between $S$. equi spp. zooepidemicus cultured in THB medium alone and S. equi spp. zooepidemicus cultured in the THB medium supplemented with non-immunized mouse serum $(P>0.05)$. These results 


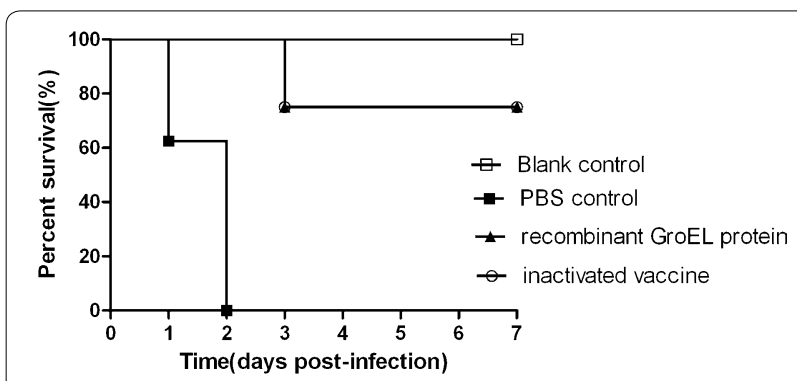

Figure 4 Protective efficacy by vaccination in mice. Mice were injected with S. equi spp. zooepidemicus ATCC35246 and mortality was recorded daily for 7 days. Mice in the non-immunized group died 24 to $48 \mathrm{~h}$ after the challenge, and the mortality rate reached $100 \%$. Mice in the groups immunized with rGroEL or the inactivated S. equi spp. zooepidemicus vaccine died 48 to $72 \mathrm{~h}$ after the challenge with $\mathrm{S}$. equi spp. zooepidemicus and $75 \%$ survived 7 days post-infection for these two groups. Significant differences in survival were noted, log rank test, $P<0.05$. Compared with the non-immunized group, the mice in the groups immunized with rGroEL or the inactivated S. equi spp. zooepidemicus vaccine had higher survival rates $(P<0.05)$.

suggest that other components found in blood serum had no inhibitory effect on S. equi spp. zooepidemicus growth or biofilm production and that the anti-rGroEL antibody had an inhibitory effect on biofilm production (Figure 5).

\section{Discussion}

S. equi spp. zooepidemicus is associated with a wide variety of infections in many animal species, including

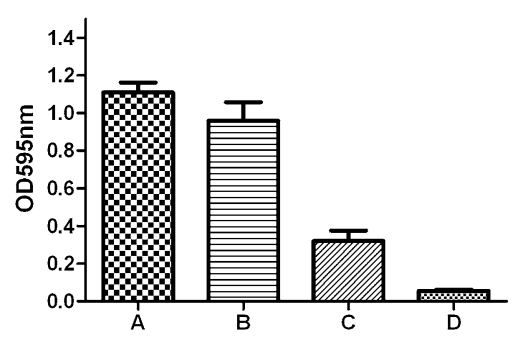

Figure 5 Quantitative microtiter plate assay for biofilm production by S. equi spp. zooepidemicus. Biofilm formation was evaluated by monitoring the $A_{595 \mathrm{~nm}}$ following crystal violet staining of bacterial cultures. The columns represent the means and standard deviations of three experiments. A Biofilm formation in THB medium (control); B Biofilm formation in THB medium supplemented with normal mouse serum; C Biofilm formation in THB medium supplemented with mouse rGroEL-antiserum; D THB medium. Student's t-test was performed for the statistical significance analysis. Biofilm formation in THB medium supplemented with anti-rGroEL serum (C) was significantly lower $(P<0.01)$ than that of S. equi spp. zooepidemicus cultured in THB medium alone $(\mathbf{A})$ or in THB medium supplemented with non-immunized pathogen-free mouse serum (B). No significant differences were found between S. equi spp. zooepidemicus cultured in THB medium alone and S. equi spp. zooepidemicus cultured in the THB medium supplemented with non-immunized mouse serum $(P>0.05)$. horses, cows, swine, sheep, and dogs [19]. More specifically, S. equi spp. zooepidemicus is the main bacterial swine pathogen in China [1]. It could also be detected in pigs without apparent clinical signs or symptoms of infection. It has also been reported to cause zoonotic infections in humans [20]. The disease is generally sporadic and outbreaks are usually of short duration; however in large herds the bacteria may be present for longer periods [21-24]. Previous studies have shown that S. equi spp. zooepidemicus can be isolated from previously described outbreaks in pig herds, thus suggesting that some S. equi spp. zooepidemicus strains can form dormant or persister cells that can be reactivated and cause infections $[23,25]$.

In other bacterial species, biofilms have been shown to play a key role in causing chronic infections [26, 27]. Grenier et al. [28] reported that a Streptococcus suis serotype 2 strain isolated from a case of meningitis in pigs could form a dense biofilm and suggested a correlation between biofilm formation and the establishment of infection. Bacteria with a capacity to colonize the host by forming biofilms have significant advantages in establishing persistent infections [29]. S. equi spp. zooepidemicus has been previously reported to form biofilms; differences in gene expression and protein profiles for planktonic- and biofilm-grown bacteria were demonstrated [5]. Attempts to efficiently control S. equi spp. zooepidemicus infections are complicated due to a lack of thorough knowledge on protective bacterial antigens. Therefore, it is important to identify antigenic components of interest for the development of a potential vaccine candidate against $S$. equi spp. zooepidemicus infections.

In this study, we used an immunoproteomic approach to search for immunoreactive S. equi spp. zooepidemicus proteins in biofilms. While previous studies have assessed the immunogenicity of $S$. equi spp. zooepidemicus using planktonic cells [3, 4], our report is the first to describe biofilm-specific proteins recognized by host antibodies. We identified seventeen immunoreactive proteins, of which nine were present in both planktonic and biofilm-grown bacteria. To our knowledge, this is the first time that the other eight immunoreactive proteins have been described in S. equi spp. zooepidemicus. The common immunoreactive proteins may be promising candidates for the development of a vaccine aimed at preventing both biofilm formation and acute $S$. equi spp. zooepidemicus infections. In this regard, the GroEL protein may be of high interest.

GroEL belongs to the chaperonin family of molecular chaperones. It is essential for biofilm formation in the Gram negative actinobacterium Haemophilus influenzae [18]. GroEL in Streptococcus pneumoniae can induce high antibody titers, promote lymphocyte proliferation, and induce both humoral and cell-mediated immune 
responses, suggesting that it may represent a protein of therapeutic interest [30]. A number of immunoproteomic studies have shown that GroEL is an immunogenic protein in a wide variety of bacteria, including Helicobacter pylori, Riemerella anatipestifer, Brucella spp., and E. coli [31-36]. Immunization with GroEL has also been shown to induce protection in a number of infection models. For instance, a significantly higher antibody titer was produced when mice were immunized with GroEL from Streptococcus pneumoniae, providing $50 \%$ protection rate against lethal infections [30]. In BALB/c mice, passive immunization with GroEL from Bacillus anthracis conferred $100 \%$ protection against Bacillus anthracis infections [37].

A mouse model was used to investigate the immunogenicity and protective immune response of the recombinant S. equi spp. zooepidemicus GroEL protein. Based on the ELISA results, the protein elicited high antibody titers. Animal challenge experiments with the S. equi spp. zooepidemicus ATCC 35246 strain showed that $75 \%$ of the immunized mice were protected, a protection rate comparable to that obtained with inactivated bacterins.

GroEL is present in several bacterial fractions, including the cytosol, cell membrane, and extracellular material [38]. It is also the compound in the biofilm supernatant that is responsible for the anti-inflammatory effect of suppressing TNF- $\alpha$ production in Lactobacilli [39]. The GroEL of S. equi spp. zooepidemicus appeared to be involved in the production of biofilm since the presence of anti-rGroEL antibodies in the culture medium inhibited biofilm formation. The GroEL may thus be a promising target for the prevention/treatment of $S$. equi spp. zooepidemicus biofilm-related infections. Similar results with antibody-mediated strategies to prevent biofilm formation have been published previously. Zarankiewicz et al. [16] reported that the presence of anti-rHsp60 (GroEL) antibodies in BHI broth medium inhibited biofilm production by Histophilus somni in vitro. They observed small biofilm particles in the presence of the anti-rHsp60 antibody, whereas large biofilm complexes were produced in the control cultures.

In conclusion, the present study identified immunoreactive proteins in S. equi spp. zooepidemicus grown in biofilms for the first time. It also brought strong evidence that the rGroEL protein is a promising candidate for the development of vaccines against $S$. equi spp. zooepidemicus infections.

\section{Competing interests}

The authors declare that they have no competing interests.

\section{Author details}

${ }^{1}$ Key Lab of Animal Bacteriology, Ministry of Agriculture, Nanjing Agricultural University, Nanjing, China. ${ }^{2}$ College of Life Science, Luoyang Normal University, Luoyang, China. ${ }^{3}$ College of Animal Science and Technology, Henan
University of Science and Technology, Luoyang, China. ${ }^{4}$ Groupe de Recherche En Écologie Buccale (GREB), Faculté de Médecine Dentaire, Université Laval, Québec City, QC, Canada. ${ }^{5}$ Jiangsu Co-innovation Center for the Prevention and Control of Important Animal Infectious Diseases and Zoonoses, Yangzhou, China.

\section{Authors' contributions}

Conceived and designed the experiments: LY, YW, C-PL, H-JF. Performed the experiments: LY, ZM, H-XL, BX. Analyzed the data: LY. Contributed reagents/ materials/analysis tools: LY, ZM, H-XL. Wrote the paper: LY, YW. Critically read and corrected the manuscript: DG. All authors read and approved the final manuscript.

\section{Acknowledgements}

This study was supported by grants from the National Basic Research Program (973) of China (2012CB518804), the National Natural Science Foundation of China $(31172319,31272581,31201910)$, the Jiangsu Agricultural Science and Technology Innovation Fund (CX(12)3078), the Key Technology Program of Jiangsu Province (BE2013433), the Priority Academic Program Development of Jiangsu Higher Education Institutions (PAPD), the Program for Science and Technology Innovation Talents in Universities of Henan Province (14HASTIT024), the Science and Technology Research Foundation of the Henan Province Educational Committee (13A230261, 14A230003), the Science and Technology Development Project of Henan Province (142102310297), and the Foundation for University Key Teachers of the Ministry of Education of Henan Province (2013GGJS-068)

Received: 19 October 2015 Accepted: 11 February 2016

Published online: 18 April 2016

References

1. Feng ZG, Hu JS (1977) Outbreak of swine streptococcosis in Sichuan province and identification of pathogen. Anim Husbandry Vet Med Lett 2:7-12

2. Wei Z, Fu Q, Liu X, Xiao P, Lu Z, Chen Y (2012) Identification of Streptococcus equi ssp. zooepidemicus surface associated proteins by enzymatic shaving. Vet Microbiol 159:519-525

3. Mao Y, Fan H, Lu C (2008) Immunoproteomic assay of extracellular proteins in Streptococcus equi ssp. zooepidemicus. FEMS Microbiol Lett 286:103-109

4. Mao Y, Fan H-J, Zhou Y-H, Lu C-P (2011) Immunoproteomic assay of antigenic surface proteins in Streptococcus equi ssp. zooepidemicus. Agr Sci China 10:1096-1105

5. Yi L, Wang Y, Ma Z, Zhang H, Li Y, Zheng JX, Yang YC, Fan HJ, Lu CP (2014) Biofilm formation of Streptococcus equi ssp. zooepidemicus and comparative proteomic analysis of biofilm and planktonic cells. Curr Microbiol 69:227-233

6. Jefferson KK, Cerca N (2006) Bacterial-bacterial cell interactions in biofilms: detection of polysaccharide intercellular adhesins by blotting and confocal microscopy. Methods Mol Biol 341:119-126

7. Shahrooei M, Hira V, Khodaparast L, Stijlemans B, Kucharikova S, Burghout P, Hermans PW, Van Eldere J (2012) Vaccination with SesC decreases Staphylococcus epidermidis biofilm formation. Infect Immun 80:3660-3668

8. Zhai Z, Li X, Xiao X, Yu J, Chen M, Yu Y, Wu G, Li Y, Ye L, Yao H, Lu C, Zhang W (2013) Immunoproteomics selection of cross-protective vaccine candidates from Riemerella anatipestifer serotypes 1 and 2. Vet Microbiol 162:850-857

9. Brady RA, Leid JG, Camper AK, Costerton JW, Shirtliff ME (2006) Identification of Staphylococcus aureus proteins recognized by the antibodymediated immune response to a biofilm infection. Infect Immun 74:3415-3426

10. Wang Y, Yi L, Wu Z, Shao J, Liu G, Fan H, Zhang W, Lu C (2012) Comparative proteomic analysis of Streptococcus suis biofilms and planktonic cells that identified biofilm infection-related immunogenic proteins. PLoS One 7:e33371

11. Zhang W, Lu CP (2007) Immunoproteomics of extracellular proteins of Chinese virulent strains of Streptococcus suis type 2. Proteomics 7:4468-4476 
12. Wu L, Jiang YN, Tang $Q$, Lin HX, Lu CP, Yao HC (2012) Development of an Aeromonas hydrophila recombinant extracellular protease vaccine. Microb Pathog 53:183-188

13. Jiang W, Han X, Wang Q, Li X, Yi L, Liu Y, Ding C (2014) Vibrio parahaemolyticus enolase is an adhesion-related factor that binds plasminogen and functions as a protective antigen. Appl Microbiol Biotechnol 98:4937-4948

14. Fan H-J, Tang F-Y, Mao Y, Lu C-P (2009) Virulence and antigenicity of the szp-gene deleted Streptococcus equi ssp. zooepidemicus mutant in mice. Vaccine 27:56-61

15. Yi L, Wang Y, Ma Z, Zhang H, Li Y, Zheng JX, Yang YC, Lu CP, Fan HJ (2013) Contribution of fibronectin-binding protein to pathogenesis of Streptococcus equi ssp. zooepidemicus. Pathog Dis 67:174-183

16. Zarankiewicz T, Madej J, Galli J, Bajzert J, StefaniakT (2012) Inhibition of in vitro Histophilus somni biofilm production by recombinant Hsp60 antibodies. Pol J Vet Sci 15:373-378

17. Wang Y, Zhang W, Wu Z, Lu C (2011) Reduced virulence is an important characteristic of biofilm infection of Streptococcus suis. FEMS Microbiol Lett 316:36-43

18. Gallaher TK, Wu S, Webster P, Aguilera R (2006) Identification of biofilm proteins in non-typeable Haemophilus Influenzae. BMC Microbiol 6:65

19. Acke E, Midwinter AC, Lawrence K, Gordon SJ, Moore S, Rasiah I, Steward K, French N, Waller A (2015) Prevalence of Streptococcus dysgalactiae subsp. equisimilis and S. equi subsp. zooepidemicus in a sample of healthy dogs, cats and horses. N Z Vet J 63:265-271

20. Villamil I, Serrano M, Prieto E (2015) Streptococcus equi subsp. Zooepidemicus endocarditis. Rev Chilena Infectol 32:240-241 (in Spanish)

21. Chalker VJ, Brooks HW, Brownlie J (2003) The association of Streptococcus equi subsp. zooepidemicus with canine infectious respiratory disease. Vet Microbiol 95:149-156

22. Pesavento PA, Hurley KF, Bannasch MJ, Artiushin S, Timoney JF (2008) A clonal outbreak of acute fatal hemorrhagic pneumonia in intensively housed (shelter) dogs caused by Streptococcus equi subsp. zooepidemicus. Vet Pathol 45:51-53

23. Salasia SI, Wibawan IW, Pasaribu FH, Abdulmawjood A, Lammler C (2004) Persistent occurrence of a single Streptococcus equi subsp. zooepidemicus clone in the pig and monkey population in Indonesia. J Vet Sci 5:263-265

24. Mellata M, Dho-Moulin M, Dozois CM, Curtiss R 3rd, Brown PK, Arne P, Bree A, Desautels C, Fairbrother JM (2003) Role of virulence factors in resistance of avian pathogenic Escherichia coli to serum and in pathogenicity. Infect Immun 71:536-540

25. Petersen MR, Skive B, Christoffersen M, Lu K, Nielsen JM, Troedsson MH, Bojesen AM (2015) Activation of persistent Streptococcus equi subspecies zooepidemicus in mares with subclinical endometritis. Vet Microbiol 179:119-125

26. Chen L, Wen YM (2011) The role of bacterial biofilm in persistent infections and control strategies. Int J Oral Sci 3:66-73
27. Woo JK, Webb JS, Kirov SM, Kjelleberg S, Rice SA (2012) Biofilm dispersal cells of a cystic fibrosis Pseudomonas aeruginosa isolate exhibit variability in functional traits likely to contribute to persistent infection. FEMS Immunol Med Microbiol 66:251-264

28. Grenier D, Grignon L, Gottschalk M (2009) Characterisation of biofilm formation by a Streptococcus suis meningitis isolate. Vet J 179:292-295

29. Falkinham JO 3rd (2007) Growth in catheter biofilms and antibiotic resistance of Mycobacterium avium. J Med Microbiol 56:250-254

30. Khan MN, Shukla D, Bansal A, Mustoori S, Ilavazhagan G (2009) Immunogenicity and protective efficacy of GroEL (hsp60) of Streptococcus pneumoniae against lethal infection in mice. FEMS Immunol Med Microbiol 56:56-62

31. Krah A, Schmidt F, Becher D, Schmid M, Albrecht D, Rack A, Buttner K, Jungblut PR (2003) Analysis of automatically generated peptide mass fingerprints of cellular proteins and antigens from Helicobacter pylori 26695 separated by two-dimensional electrophoresis. Mol Cell Proteomics 2:1271-1283

32. Krah A, Miehlke S, Pleissner KP, Zimny-Arndt U, Kirsch C, Lehn N, Meyer TF, Jungblut PR, Aebischer T (2004) Identification of candidate antigens for serologic detection of Helicobacter pylori-infected patients with gastric carcinoma. Int J Cancer 108:456-463

33. Hu Q, Ding C, Tu J, Wang X, Han X, Duan Y, Yu S (2012) Immunoproteomics analysis of whole cell bacterial proteins of Riemerella anatipestifer. Vet Microbiol 157:428-438

34. Abbady AQ, Al-Daoude A, Al-Mariri A, Zarkawi M, Muyldermans S (2012) Chaperonin GroEL a Brucella immunodominant antigen identified using Nanobody and MALDI-TOF-MS technologies. Vet Immunol Immunopathol 146:254-263

35. Bao Y, Zhai Z, Wang S, Ma J, Zhang W, Lu C (2013) Chaperonin GroEL: a novel phylogenetically conserved protein with strong immunoreactivity of Avian Pathogenic Escherichia coli isolates from duck identified by immunoproteomics. Vaccine 31:2947-2953

36. Han X, Hu Q, Ding S, Chen W, Ding C, He L, Wang X, Ding J, Yu S (2012) Identification and immunological characteristics of chaperonin GroEL in Riemerella anatipestifer. Appl Microbiol Biotechnol 93:1197-1205

37. Sinha K, Bhatnagar R (2010) GroEL provides protection against Bacillus anthracis infection in BALB/c mice. Mol Immunol 48:264-271

38. Skar CK, Kruger PG, Bakken V (2003) Characterisation and subcellular localisation of the GroEL-like and DnaK-like proteins isolated from Fusobacterium nucleatum ATCC 10953. Anaerobe 9:305-312

39. Rieu A, Aoudia N, Jego G, Chluba J, Yousfi N, Briandet R, Deschamps J, Gasquet B, Monedero V, Garrido C, Guzzo J (2014) The biofilm mode of life boosts the anti-inflammatory properties of Lactobacillus. Cell Microbiol 16:1836-1853

\section{Submit your next manuscript to BioMed Central and we will help you at every step:}

- We accept pre-submission inquiries

- Our selector tool helps you to find the most relevant journal

- We provide round the clock customer support

- Convenient online submission

- Thorough peer review

- Inclusion in PubMed and all major indexing services

- Maximum visibility for your research

Submit your manuscript at www.biomedcentral com/submit 